\title{
Computed tomography features of liparitosis: a pneumoconiosis due to amorphous silica
}

\author{
S. Mazziotti*, M. Gaeta*, C. Costa ${ }^{\#}$, G. Ascenti*, L. Barbaro Martino\#, G. Spatari\#, N. Settineri*, M. Barbaro ${ }^{\#}$
}

Computed tomography features of liparitosis: a pneumoconiosis due to amorphous silica. S. Mazziotti, M. Gaeta, C. Costa, G. Ascenti, L. Barbaro Martino, G. Spatari, N. Settineri, M. Barbaro. (C) ERS Journals Ltd 2004.

ABSTRACT: The purpose of this study was to describe the radiological features of pleural, parenchymal and mediastinal damage due to occupational inhalation of pumice.

In total, 31 workers were evaluated for annual follow-up. Spiral computed tomography (CT) was obtained in the three patients with evident parenchymal abnormalities at chest radiograph. High-resolution computed tomography (HRCT) was performed in the remaining 28 subjects with no radiographical evidence of pleuropulmonary damage or evidence of pleural plaques, in order to exclude parenchymal involvement. Pumice samples were analysed by radiograph diffraction and scanning electron microscopy.

Spiral CT showed parahilar conglomerate masses containing fine reticular calcifications in three of $31(9.7 \%)$ patients. HRCT showed several bilateral pleural plaques, without any parenchymal lesion in eight of $28(28.6 \%)$ cases. Statistical analysis indicated correlation between $\mathrm{CT}$ findings and pulmonary function, as well as exposure to pumice. The analysis of pumice excluded the presence of asbestos.

The findings from this study suggest that pleural plaque may be the only sign of disease in patients with a history of pumice inhalation. However, conglomerate parahilar masses are probably due to exposure to massive amounts of heated pumice, and seem to have different pathogenesis and histopathological characteristics to silicosis. Eur Respir J 2004; 23: 208-213.
*Dept of Radiological Sciences and ${ }^{\#}$ Dept of Social Medicine, Occupational Medicine Section, University of Messina, Messina, Italy.

Correspondence: S. Mazziotti

Via Consolare Pompea, 45

98168 Messina

Italy

Fax: 390902213720

E-mail:smazziotti@unime.it

Keywords: Amorphous silica computed tomography lung pleura pumice

Received: March 162003

Accepted after revision: August 282003
Pulmonary silicosis is a well-described disease caused by inhalation of crystalline silica. It is typically characterised by multiple discrete parenchymal nodules usually measuring a few millimeters in diameter, with upper lobe predominance. In advanced cases, pleural surfaces may also be fibrotic, with silicotic nodules and rarely, diffuse plaques.

The description of pneumoconioses due to amorphous silica is undoubtedly less defined. One of these, named "liparitosis" by CASTRONOvo [1], is related to the inhalation of pumice powder extracted in the island of Lipari (Aeolian Archipelago, Sicily). Despite its low incidence due to localised exposure, liparitosis deserves a certain interest as it can be considered representative of pneumoconioses derived by amorphous silica compounds, including diatomite, Fuller's earth and above all, artificial amorphous silica, the industrial manufacturing of which is extremely widespread. Artificial amorphous silica is utilised for the production of paints, adhesives, sealants, insulators, mineral oils, electrical material, inks, toners, plastics, paper, films, cosmetics, pharmaceuticals, and toothpaste. Pumice itself is widely used for the production of concrete, soils, detergents, abrasives, filters, toothpaste, electrical material and for the "stone-washing" of jeans.

Pumice is a natural, complex silicate containing $\sim 70 \%$ silica $\left(\mathrm{SiO}_{2}\right)$ in which other oxides are dissolved (aluminium $(\sim 12 \%)$, titanium $(\sim 0.1 \%)$, iron $(\sim 2 \%)$, manganese $(\sim 0.1 \%)$, sodium $(\sim 3.6 \%)$ and potassium $(\sim 4.5 \%)$ oxides) forming the relative silicates [2]. The plentiful presence of ions does not allow the formation of a crystalline reticulum, therefore pumice has an amorphous but typically not fibrous structure. Pumice quarries also exist in the USA, Greece, Turkey and Japan.
Two different presentations of liparitosis have been described in the past [3]; a massive nodular sclerosis limited to basal lung and an irregular diffuse fibrosis of the middle and basal lung. Recently, two cases of primary pleural involvement after exposure to amorphous silicates have been described [4].

The purpose of this study was to describe the radiological features of pleural, parenchymal and mediastinal damage due to inhalation of pumice powder in a wider study population of exposed workers.

\section{Materials and methods}

From June 1999 to September 2002, 31 subjects occupationally exposed to pumice in the island of Lipari were evaluated for annual follow-up. All subjects were male and aged $61.02 \pm 10.58$ (mean $\pm \mathrm{SD})$ yrs. Occupational exposure to pumice was evaluated by recording job title and description, duration of exposure (number of years working in the quarry) and latency of symptoms since initial exposure. The subjects had worked in the quarry of Lipari for $24.86 \pm 11.81$ (mean \pm SD) yrs. None gave a history of occupational or domestic exposure (either past or present) to asbestos and mineral dusts other than pumice.

Subjects were identified as smokers, exsmokers and nonsmokers. Each subject underwent a clinical examination, followed by spirometry and standard chest radiograph. The International Labor Office (ILO) method was used for assessment of small parenchymal opacities profusion.

Pulmonary function tests were carried out with spirometry 
equipment, with the performance meeting American Thoracic Society recommendations. Results were expressed as percentages of predicted normal values for correlation analysis.

On the basis of standard chest radiograph findings, spiral computed tomography (CT) of the chest was obtained in the patients with evident parenchymal abnormalities, with a slice thickness of $8 \mathrm{~mm}$ and a table increment of $10 \mathrm{~mm}$, using settings of $140 \mathrm{kV}$ and $159 \mathrm{~mA}$.

High Resolution Computed Tomography (HRCT) was performed through the entire chest in the remaining patients with no sign of pleuro-pulmonary damage or evidence of pleural plaques, in order to exclude parenchymal involvement. HRCT images of $1 \mathrm{~mm}$ slice thickness were obtained at $10 \mathrm{~mm}$ intervals, using settings of $140 \mathrm{kV}, 111 \mathrm{~mA}$ and a $1.5 \mathrm{~s}$ acquisition time. This different assignment of patients was done on a prospective basis, since spiral CT permits a complete coverage of lung parenchyma necessary to evaluate the extension of lung damage in patients with demonstrated lung disease. However, HRCT has been shown to permit diagnosis of lung disease in patients who have questionable or negative plain radiographical films associated with chronic disease [5].

All CT studies were performed with a Somatom Plus 4 (Siemens, Enlargen, Germany) without injection of contrast material. All CT scans were obtained during suspended deep inspiration in supine position. Both HRCT and Spiral-CT images were photographed at two window settings, appropriate for viewing the entire lung parenchyma, pleura and mediastinum.

Annual follow-up examinations were obtained for all patients for 3 yrs. Both chest radiograph films and CT images were evaluated by two skilled readers, a radiologist and a pneumologist, with $>10$ yrs experience in using the ILO classification. Discrepancies were resolved by consensus.

Only two of the subjects accepted a CT guided needle biopsy and only one patient with pleural plaques underwent pleuroscopy. Therefore, in order to correlate the radiological in vivo findings of parahilar masses with the underlying pathological alterations, the authors retrieved and reviewed radiological and histological material obtained by autopsy performed between 1950 and 1955 in two patients with liparitosis, whose radiological studies showed the presence of parahilar masses comparable to those observed in patients in the current study.

Analysis of variance was used to determine the significant differences between group mean values of pulmonary function, smoking habits and years of exposure to pumice powder, using CT findings as a variable factor. Moreover, the nonparametric Spearman rank test was used to assess the correlation between CT findings and the measurements of pulmonary function, number of cigarettes per day and years of exposure to pumice powder.

Pumice powder samples collected in the quarry of Lipari were analysed by means of X-ray diffraction techniques (XRD with a Siemens R2000 diffractometer equipped with a graphite monochromator) at $30 \mathrm{~mA}$ and $40 \mathrm{kV}$ working conditions, in the range of 2-75 degrees $2 \theta$. All experiments were carried out with $\mathrm{NaF}$ as an internal standard for calibration. NaF peaks were after omitted in the graphics.

Scanning electron microscopy (SEM) was performed on a Leo S420 (Leica-Cambridge Instruments, Cambridge, UK) coupled with an energy dispersive system (EDX) (Oxford Link ISIS series; Oxford Instruments, Witney, UK) 300 with SiLi detector (Oxford Instruments), operating at $>156 \mathrm{eV}$ (SEM+EDX).

\section{Results}

The most common symptoms reported by clinical examination were dyspnoea, cough and asthenia. Patients were divided in three groups on the basis of a standard chest radiograph. Group 1 consisted of 22 of 31 subjects (71\%) with no radiographical alteration (ILO 0/0-1). Group 2 was composed of six of 31 patients $(19.3 \%)$ with pleural plaques (ILO a-c/1-3). Group 3 consisted of three of 31 patients $(9.7 \%)$ with evidence of parahilar masses (ILO A-C). Workers belonging to groups 1 and 2 were employed in excavation, mills, sieves and transportation, while those classified as group 3 had worked with desiccation ovens.

The cohort of this study was referred to the authors' institution for annual medical surveillance due to occupational exposure to siliceous dust. Only $29 \%$ of these subjects had pathological findings on the radiograph. However, these negative cases were not excluded from the study population, in order to give a representative and up to date view of the natural history of this disease.

Mean \pm SD of functional parameters derived from spirometry, years of exposure to pumice powder and number of cigarettes per day are reported in table 1 . No exsmokers were present in the series.

HRCT, carried out on all 28 workers of groups 1 and 2, showed pleural plaques in eight of $28(28.6 \%)$ cases, confirming the presence of several bilateral plaques without any parenchymal lesion in six cases in group 2 (fig. 1) and revealing pleural alterations in two cases of group 1 not evidenced by standard chest radiograph. Plaques were $<1 \mathrm{~cm}$ thick, the largest measuring $\sim 7 \times 5 \mathrm{~cm}$ and the smallest $\sim 1 \times 1 \mathrm{~cm}$. Fibroscopy and CT guided needle biopsy, carried out in patients one and two respectively in group 2, demonstrated the fibrotic nature of pleural plaques. Fibrotic plaques involved both parietal and visceral pleura, which cannot be separated. No asbestos bodies were found in biopsy specimens.

In all patients, no growth of pleural plaques was seen at annual follow-up CT for up to 3 yrs.

Spiral-CT, performed in the three patients from group 3, showed parahilar conglomerate masses containing fine reticular calcifications. This pattern of calcification was quite different from calcification seen in conglomerate masses of silicosis [6]. Calcification was also present in mediastinal lymphnodes, but the authors did not see eggshell calcification, which can be observed in 5\% cases of silicosis and is almost patognomonic of this entity [7]. No significant distortion of lung parenchyma around parahilar masses could be seen. However, slight bronchial distortion was demonstrated in patients with masses. In one patient from group 3, pulmonary alterations were associated with focal pleural thickening (fig. 2). Pulmonary nodules typical of silicosis by crystalline silica were never seen in the patients from the current study [8].

The correlation between radiological findings of parahilar conglomerated masses with the underlying pathologic alterations found in retrieved histological material allowed the

Table 1.-Pulmonary function, exposure time and smoking habits in the three groups, identified on the basis of standard chest radiograph

\begin{tabular}{lclcc}
\hline & Group 1 & Group 2 & Group 3 & p-value \\
\hline FVC \% & $90.6 \pm 9.1$ & $83.5 \pm 10.4$ & $64.0 \pm 6.2$ & $<0.001$ \\
FEV 1 \% & $94.6 \pm 15.4$ & $77.8 \pm 9.1$ & $68.7 \pm 4.2$ & 0.004 \\
FEF 25-75 \% & $111.1 \pm 30.7$ & $80.0 \pm 5.5$ & $74.0 \pm 4.0$ & 0.014 \\
Exposure yrs & $15.0 \pm 7.4$ & $31.2 \pm 3.0$ & $31.3 \pm 3.5$ & $<0.001$ \\
Cigarettes day & $10.9 \pm 11.2$ & $12.2 \pm 12.4$ & $2.7 \pm 3.1$ & NS \\
\hline
\end{tabular}

Data are presented as mean \pm SD unless otherwise stated. FVC: forced vital capacity; FEV1: forced expiratory volume in one second; FEF25-75: forced mid-expiratory flow; NS: nonsignificant. 

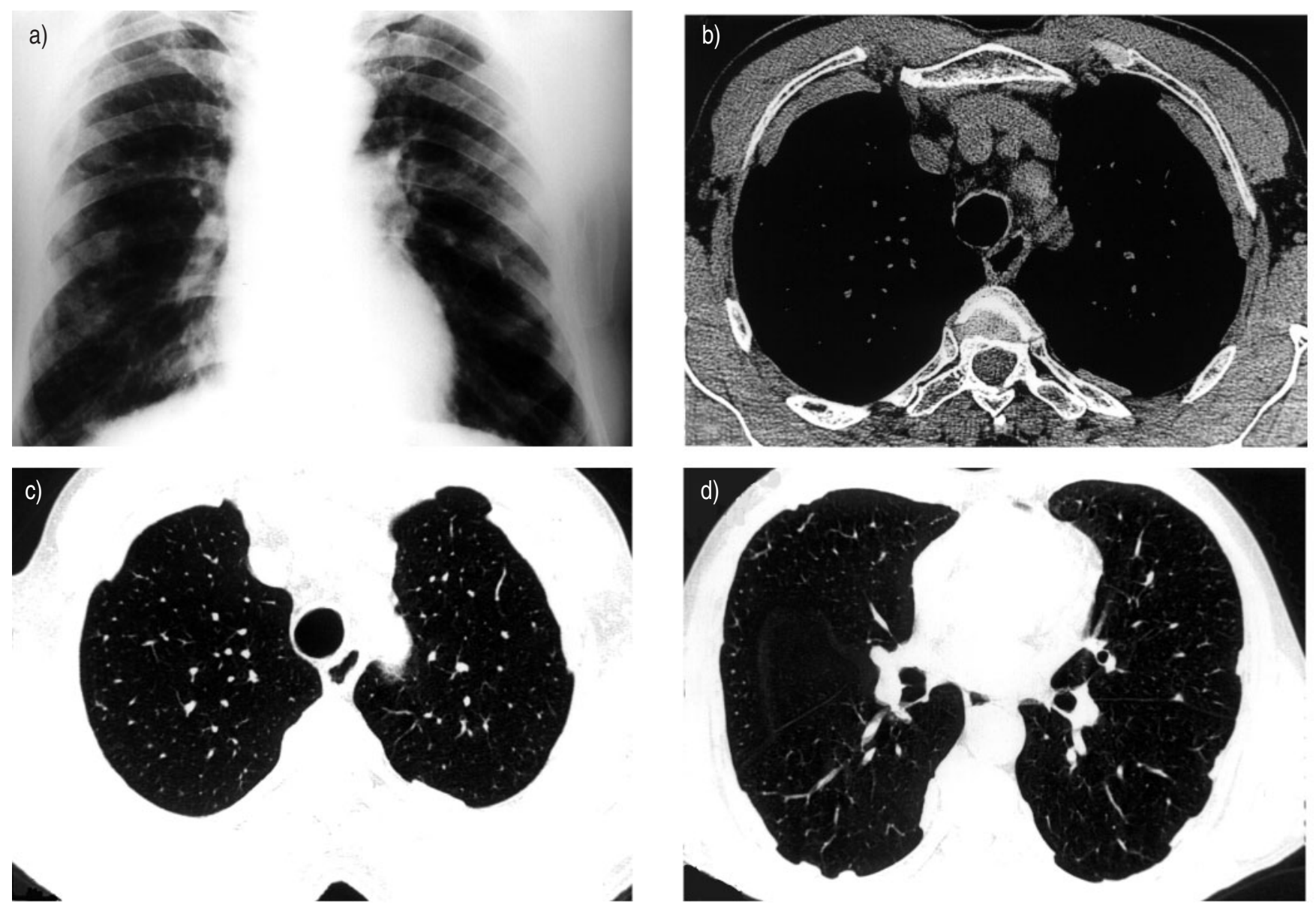

Fig. 1.-A 65-yr-old male occupationally exposed to pumice in the pretechnological period. a) The chest radiograph demonstrates multiple and bilateral pleural plaques. Transverse 1-mm thick high resolution computed tomography sections visualised with b) mediastinal and lung settings at c) higher and d) lower levels confirm the presence of several pleural plaques localised bilaterally, without any parenchymal lesion.

authors to describe the masses as fibrotic parenchymal areas including lymph nodes. Massive deposition of dust in the peribronchial and perivasal connective tissue and, in later phase, presence of macrophages and fibrosis in the parahilar lung were visible (fig. 3).

Statistical analysis indicated that pulmonary functional parameters differed significantly between the three groups (table 1). It also showed a significant difference in exposure time, but not in cigarette smoking between the groups.

The Spearman test indicated that there was significant correlation between CT findings in groups 1, 2 and 3 (none, pleural plaques, parahilar conglomerated masses) and pulmonary functional parameters, as well as in the number of years of exposure to pumice powder. Conversely, smoking habits did not appear to correlate with CT findings (table 2).

$\mathrm{X}$-ray diffraction data for pumice powder in the examined specimens chemically identified them as $\mathrm{SiO}_{2}$, despite a tendency to initial crystallisation (following the increasing peaks, not clearly defined, in the region 20-30 degrees 2 $\theta$ ), analysis revealed a diffraction pattern pointing mainly to amorphous silica (fig. 4a). Morphological examination at SEM showed irregular particles, similar to broken glass, with
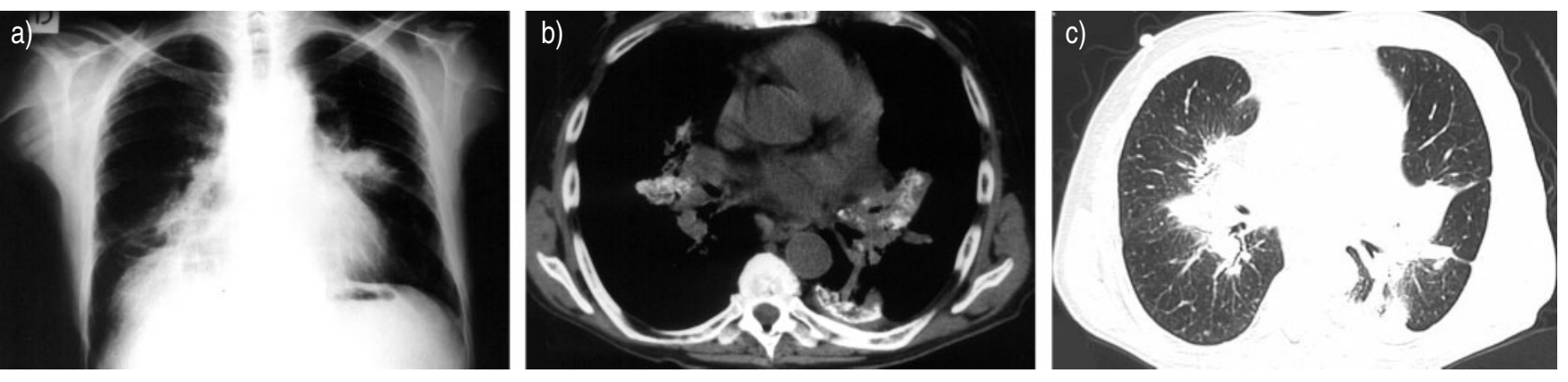

Fig. 2. - A 69-yr-old male occupationally exposed to massive amounts of heated pumice in the pretechnological period. a) Chest radiograph shows irregular masses in the parahilar regions. b) Transverse spiral computed tomography (CT) scan through the subcarinal region reveals fine reticular calcification of the parahilar masses. A pleural plaque is also visible on the left. A slight diffuse calcification is apparent in the subcarinal lymph node. c) CT scan viewed at lung window setting shows parahilar masses but not significant distortion of the peripheral lung parenchyma. A slight degree of distortion of some segmental bronchi is apparent. 

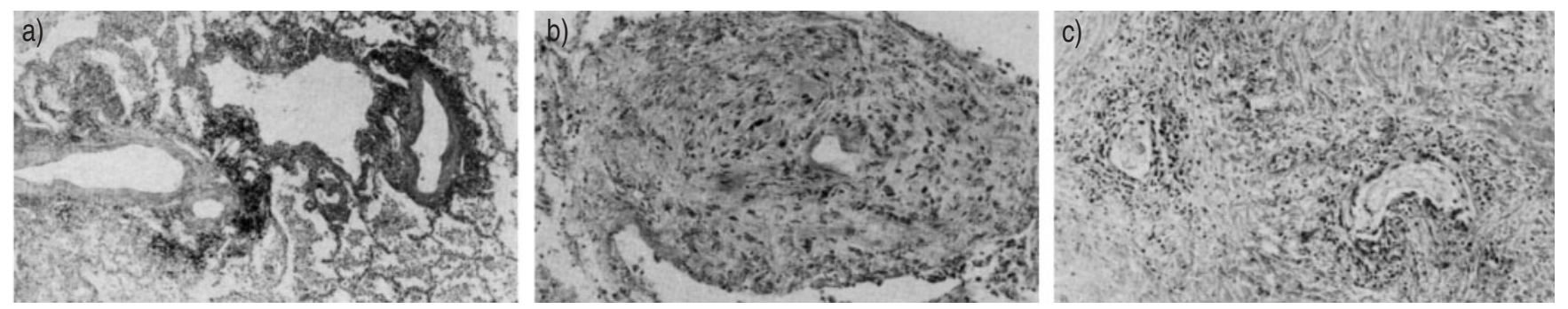

Fig. 3.-Microphotographs of lung autoptic specimens (haematoxylin and eosin staining). a) Pumice powder is deposited in the peribronchial and perivasal connective tissue. b) "Nodular" structure with a rich population of macrophages surrounding a small vasal lumen. c) Massive sclerosis with a concentric disposition around the venules.

Table 2. - Correlation between CT findings and pulmonary function parameters, number of cigarettes per day and years of exposure to pumice

\begin{tabular}{lr}
\hline & p-value \\
\hline FVC \% & 0.005 \\
FEV1 \% & 0.001 \\
FEF25-75 \% & 0.001 \\
Exposure yrs & $<0.001$ \\
Cigarettes day & NS \\
\hline
\end{tabular}

Data show the results of the two-tailed Spearman correlation coefficient. FVC: forced vital capacity; FEV1: forced expiratory volume in one second; FEF25-75: forced mid-expiratory flow; NS: nonsignificant.

sharp and pointed angles. Particles were often oblong, with one dimension prevailing over the others, but never fibrous (fig. 4b), thus excluding the presence of asbestos. Therefore, neither quartz nor asbestos should be considered responsible for the parenchymal and pleural lesions observed.

\section{Discussion}

One of the most common diseases due to occupational exposure to inhaled substances is pulmonary silicosis. It is a typical fibrogenic, nodular pneumoconiosis determined by quartz (i.e. crystalline, free silica) and with well known clinical, radiological and histopathological features. However, the industrial use of amorphous silica and silicates has increased and although noncrystalline and nonfibrous silica is considered "less toxic" due to its minor fibrogenicity, its toxic potential should not be underestimated $[9,10]$.

A non-nodular pulmonary fibrosis has been described, among the others, in workers exposed to diatomite [11, 12] and Fuller's earth [13]. The current study describes the characteristics of liparitosis, another rare pneumoconiosis due to amorphous silica.

Liparitosis is characterised by a chronic evolution of 20-30 yrs. Clinically it is almost silent, vaguely simulating a catarrhal bronchitis. Some peculiar characteristics have been described for this disease, suggesting it is a defined pneumoconiosis. From a radiological standpoint, CASTRONOVO [1] described the progression of this illness from a fine reticulation to a later stage characterised by mass-like fibrosis. In a previous report [4] the CT findings in two cases of primary pleural lesions due to pumice inhalation have been described.

Today, probably thanks to the technological improvement of extraction and processing procedures, the massive form is still visible only in a few workers of $>20$ yrs of working age. On the basis of the substantial improvement of the processing plant in 1985, the period before 1985 can be defined as pretechnological and the following period as technological.
Respirable dust mean levels were $6.8 \mathrm{mg} \cdot \mathrm{m}^{-3}$ in 1981 and $1.9 \mathrm{mg} \cdot \mathrm{m}^{-3}$ in 1997 . Excavation became mechanised, aspiration was applied to mills and sieves, desiccation was performed in sealed apparatus and transportation of sacks containing the powder was automated. All workers belonging to the technological period were classified in group 1, presenting no sign of pleural and pulmonary damage at standard chest radiograph. Also, four subjects with $>20$ yrs of working age, thus belonging to pretechnological period, showed no pulmonary damage at chest radiograph. However, two showed small pleural plaques at HRCT. In group 2, the presence of pleural plaques was evident on a standard chest radiograph. HRCT confirmed this finding, allowing a more accurate evaluation of the number and extension of plaques. HRCT also demonstrated the absence of parenchymal alterations, which seems to differentiate this pneumoconiosis from silicosis. All these patients belonged to the pretechnological period, like those classified as group 3. However, patients from group 3 were exposed, in the pretechnological period, to massive amounts of heated pumice. Patients from this group showed bilateral parahilar masses. The pulmonary mass characterised by fine reticular calcifications showed by CT allowed the diagnosis of neoplastic expansive process to be excluded. To the authors' knowledge, this pattern of calcification has not been described in patients with pneumoconiosis caused by crystalline silica $[6,7]$. It might be postulated that heating of pumice for desiccation might convert its structure from amorphous into crystalline. However, it is improbable that temperatures reached in the desiccation ovens could be high enough to determine this transformation; more probably, loss of humidity reduced the aerodynamic diameter of dust particles allowing them to penetrate more deeply into the airways.

Although in the radiological literature high density deposition within fibrotic mass is conventionally indicated as calcification, WAGNER et al. [14] have demonstrated that silicotic masses contain both mineral dust and calcium phosphate. Similarly, "radiological" calcification visible in conglomerated masses of liparitosis may correlate to the deposition of amorphous silica and probably, to some amount of calcium phosphate (fig. 3a).

Only two cases of pleural thickening have been described in patients affected by liparitosis [4]. In the current study population it was seen in as much as $29 \%$ of patients (nine of 31 ). Furthermore, in $25.8 \%$ of these patients (eight of 31 ) no associated parenchymal alteration was detectable.

The lack of radiographic findings of group 1 workers employed after 1985 tends to be attributed to the technological improvement undertaken in the caves and processing plant in that year. However, it cannot yet be excluded that it is a consequence of the shorter duration of exposure or followup. A longer observation of these subjects will surely be useful to solve this doubt.

Two previous studies analysed the effects of occupational 
exposure to amorphous silica on pulmonary function [10, 15]. These studies concluded that exposure to amorphous silica cannot be completely responsible for pulmonary functional damage, and postulate a synergism with cigarette smoking. The findings from the current study strongly support the hypothesis that exposure to pumice correlates with pulmonary function, but also highlight the difference in exposure time and pulmonary function between the three groups. The normal spirometry values of group 1 are in accordance with the shorter exposure time and with the absence of radiological findings. The same consideration can be extended to group 2 , in which the minimal deviation of functional parameters from the range of normality, despite of a long exposure time, can be explained by the absence of parenchymal damage.

The involvement of pleura in advanced cases of silicosis is well described. Pleural surfaces of the lungs appear fibrotic, with typical silicotic nodules and rarely diffuse plaques. However, only one case is described in which the disease was pleural and did not involve the underlying lung parenchyma [16]. After a deeper examination of this case report it is not possible to exclude that the patient could have actually been exposed to amorphous silica. In fact, mineralogical analysis and energy dispersive radiographic spectrometry of a tissue specimen described the presence of as much as $70 \%$ potassium aluminum silicates, aluminum silicates, calcium aluminum, and calcium and magnesium aluminum silicates. This composition may well be compatible with amorphous silica, thus explaining the peculiarity of this finding. These considerations suggest that primary, delayed pleural damage may be a peculiarity of pneumoconiosis due to pumice inhalation. However, when exposure to amorphous silica is not reported by the patient, such a findings make necessary a differential diagnosis with other pleural reactive conditions and neoplasms, including malignant mesothelioma.

The parahilar masses observed in liparitosis seem to have different hystopathological characteristics, and presumably also a different pathogenesis, from the progressive massive fibrosis typical of silicosis.

The silicotic mass derives from the confluence of sclerohyaline parenchymal nodules. Conversely, in liparitosis the salient morphological aspect is given by the massive deposition of dust in the peribronchial and perivasal connective tissue. This infiltration coating the vascular stromal structure may appear in the two dimensional histological image as a nodular structure, showing centrally located ramification of the bronchiolar vascular tree. The nodular structures are composed of macrophages, without signs of necrosis (fig. 3b). When the "nodules" become very close together they may combine in an irregular fibrous mass (fig. 3c).

The difference with silicosis may be explained by the mild cytotoxicity exerted by pumice on alveolar and interstitial macrophages and on mononuclear phagocytes; pumice powder accumulates into the cells without causing their necrosis, which is responsible of the release of interleukines and other mediators provoking fibrosclerotic reaction. Consequently, pumice powder, both free and contained in macrophages, follows an efficient lymphatic drainage towards pleural membranes and mediastinal lymphnodes determining a chronic fibrosis only after overcoming a threshold limit of dust deposit. This mechanism may explain the relative clinical mildness and long evolution of liparitosis.

The main limitation of the current study is the lack of bioptic evaluation in many patients. However, the CT findings, the follow-up radiological examinations and the bioptic results obtained in the same patients are convincing enough of the fibrotic nature of the lesions. Moreover, histological data obtained from specimens of two deceased workers confirm the peculiar characteristics of this disease.

In conclusion, the authors believe that liparitosis represents
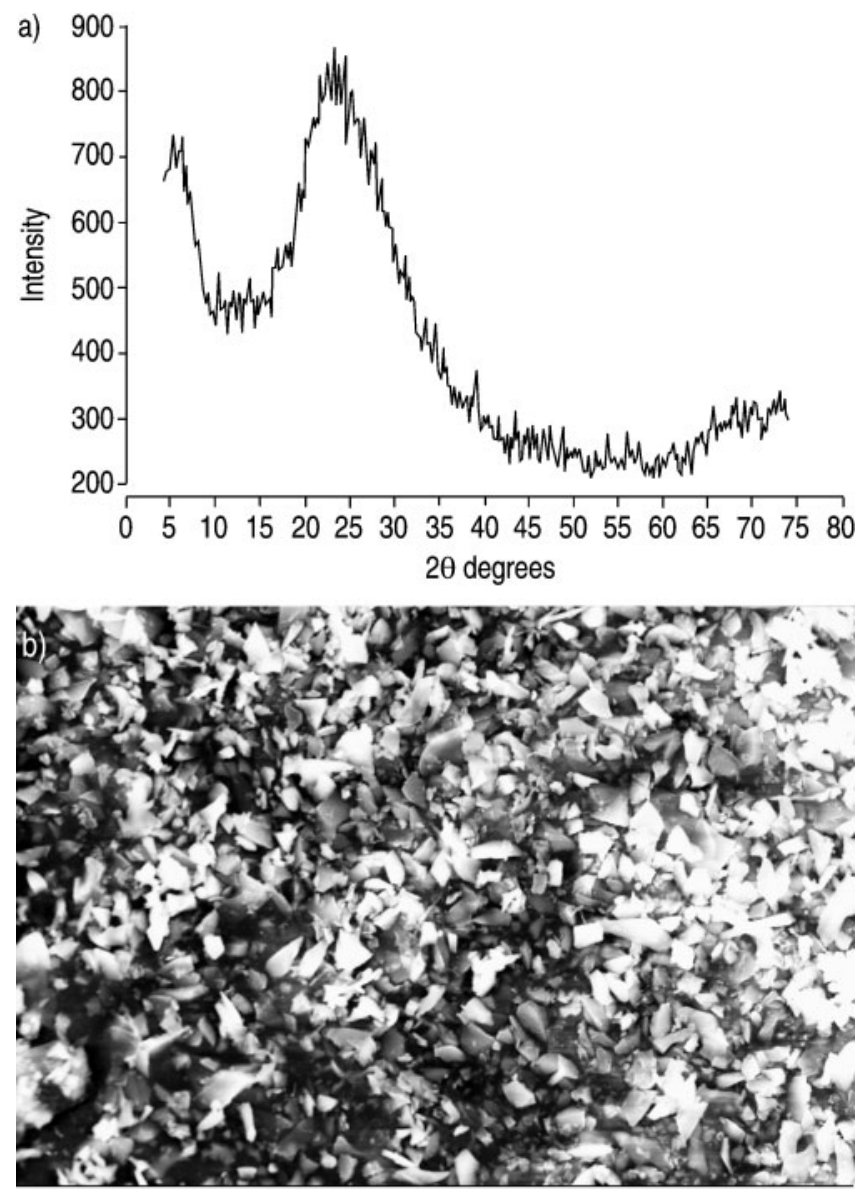

Fig. 4.-a) X-ray diffraction analysis reveals a pattern pointing mainly to amorphous silica, with a tendency to initial, not clearly defined crystallisation processes in the region 20-30 degrees $2 \theta$. b) Scanning electron microscopy morphological examination shows the presence of irregular particles, similar to broken glass with sharp and pointed angles.

a poorly known but paradigmatic disease due to amorphous silica, presenting with unique computed tomography findings. Because of wide industrial use of amorphous silica compounds, the knowledge of clinical and computed tomography features of "liparitosis" could be useful both for radiologist and pneumologist.

\section{References}

1. Castronovo E. Aspetto radiologico della pneumoconiosi da pomice (liparosi) e sua interpretazione patogenetica [Radiological features of pumice pneumoconiosis (liparitosis) and its pathogenic interpretation]. Riv Inf Mal Prof 1953; Suppl. 278-289.

2. Reference Publication from Pumex S.p.A. 3rd edn. Bergamo, Carminati Stampatore, 1985.

3. Ferrara A, Faraone G. Morfologia e patogenesi delle pneumosclerosi da pomice [Morphology and pathogenesis of pumice pneumosclerosis]. Riv Inf Mal Prof 1953; Suppl. 453-468.

4. Mazziotti S, Costa C, Ascenti G, Lamberto S, Scribano E Unusual pleural involvement after exposure to amorphous silicates (Liparitosis): report of two cases. Eur Radiol 2002; 12: $1058-1060$.

5. Webb WR, Muller NL, Naidich DP. Clinical utility of high-resolution computed tomography. In: High-resolution CT of the lung. 3rd edn. Lippincott Williams \& Wilkins, Philadelphia, 2001: pp. 569-597. 
6. Remy-Jarden M, Degreef JM, Beuscart R, et al. Coal workers pneumoconiosis: CT assessment in exposed workers and correlations with radiographic findings. Radiology 1990; 177: $363-371$.

7. Jacobson G, Felson B, Pendergrass EP, et al. Eggshell calcification in coal and metal workers. Semin Roentgenol 1967; 2: 276.

8. Webb WR, Muller NL, Naidich DP. Silicosis and coal workers pneumoconiosis. In: High-Resolution CT of the lung. 3rd edn. Lippincott Williams \& Wilkins, Philadelphia, 2001; pp. 303-309.

9. Reuzel PG, Bruijntjes JP, Feron VJ, Woutersen RA. Subchronic inhalation toxicity of amorphous silicas and quartz dust in rats. Food Chem Toxicol 1991; 29: 341-354.

10. Choudat D, Frisch C, Barrat G, el Kholti A, Conso F. Occupational exposure to amorphous silica dust and pulmonary function. Br J Ind Med 1990; 47: 763-766.
11. Miller M. Lung, pleura and mediastinum. In: Anderson WAD. Path., 6th edn. St. Louis, CV Mosby Co., 1971; pp. 929-931.

12. Abrams HK. Historical perspectives in occupational medicine. Diatomaceous hearth silicosis. Am J Ind Med 1990; 18: 591-597.

13. Gibbs AR, Pooley FD. Fuller's earth (montmorillonite) pneumoconiosis. Occup Environ Med 1994; 51: 644-646.

14. Wagner JC, Wusteman FS, Edwards JH, Hill RJ. The composition of massive lesions in coal miners. Thorax 1975; 30: $382-388$

15. Wilson RK, Stevens PM, Lovejoy HB, Bell ZG, Richie RC. Effects of chronic amorphous silica exposure on sequential pulmonary function. J Occup Med 1979; 21: 399-402.

16. Zeren EH, Colby TV, Roggli VL. Silica-induced pleural disease. An unusual case mimicking malignant mesothelioma. Chest 1997; 112: 1436-1438. 